\title{
Methods for detection of electromagnetic signals of lithospheric origin
}

\author{
Vladimir Uvarov ${ }^{1, \star}$ \\ ${ }^{1}$ Institute of Cosmophysical Researches and Radio Wave Propagation FEB RAS, \\ Paratunka, Kamchatsky Kray, Russia
}

\begin{abstract}
The paper considers physical conditions of generation and distribution of electromagnetic radiation of lithospheric origin and the peculiarities of registration based on them. Methods for measurements of seismoelectromagnetic activity, parameters and procedures, are suggested.
\end{abstract}

\section{Introduction}

The main forming forces of a solid, in particular of a rock, are the forces of electromagnetic interaction. Thus, any change of a form and of a structure of separate pieces is the change of medium electromagnetic structure. It is accompanied both by acoustic and electromagnetic disturbances. In other words, acoustic-electromagnetic emissions of the lithosphere are the manifestations of acoustic and electromagnetic disturbances of the lithosphere during its deformation under the tectonic effect.

\section{Instrumentation and methods of investigation}

Natural electromagnetic field over the Earth's surface is a mixture of fields of different origin. Its prevailing componnet is associated with atmosperic-lightning activity in the Asian, African and American Centers of the world lightning activity where 100-300 lightning strokes occur every second. The maximum field intensity at the observation site may reach units of $\mathrm{v} / \mathrm{m}$ exceeding the background vlaue by hundreds of thousands of times [1]. Radiation of lightning origin propagates mainly in the waveguide between the Earth's surface and the ionosphere where the losses are from fractions to the first units of decibels per a kilometer [2]. Thus, when observations are carried out in mid latitudes, the sources of the world lightning centres are located in the far wave zone for SLF-VLF range waves. The component of magnetospheric origin manifests itself the most during magnetic storms. Electromagnetic radiation associated with human industrial activity is significant in densely populated areas and near power lines. At present, natural electromagnetic radiation of SLF-VLF range is applied in global remote sounding carried out by international geophysical projects (TOGA, WWLLN, AWDANET) the results of which are available on their websites. However application of these data in the investigations of lithospheric radiations is problematic.

\footnotetext{
^e-mail: uvarovvnng@yandex.ru
} 
The lithospheric component is significant in the areas of increased seismic activity during its intensification. The major information on deformation processes in the crust carried over by the lowfrequency radiation of SLF-VLF range due to the absorption of high-frequency radiation by a conductive rock. When observing remote lithospheric sources, the propagation path of radiation consists of two parts, they are: from the underground source to the surface and propagation in the atmosphere from the place of radiation output to a detector. Estimates of the cone angle of electromagnetic radiation output for typical lithospheric parameters for this range of waves is within $10^{0} \div 20^{0}$ by a vertical. Thus, the output spot on the survace indicates quite accurately the source projection location. As long as the losses of electromagnetic radiation during its propagation in the Earth's crust are tens of $\mathrm{dB} / \mathrm{m}$, signals from lithospheric sources the distance to which is significantly less than the wave length are available for registration. They are located in the near wave zone.

It follows herefrom that in the scales of lithospheric source, the field of lightning origin is homogeneous, whereas the field of lithospheric origin is inhomogeneous. This main difference of statistical characteristics may be taken as the basis of the method for sorting the signals carried by these fields.

\section{Sorting of signals}

There are several ways to distinguish a LEMR signal from the background noise. Detection is based on such parameters as frequency, amplitude and polarization. Unfortunately, the features for distinguishing of LEMR signals have not been found. Physical restraint (screening) of the size of the area of radiation source and the detector is realized during the measurements in lithosphere cavities, for example in mines or wells. This approach (application of mines) is well known [3] and will not be considered thereby.

Much more effective is the compensation of a noise signal which we consider in some detail. Such an approach may be realized by two ways, scalar and vector approximation of the field.

1. During the scalar approximation of the field (registration of one parameter of the field), the compensation is based on the application of differences of spatial statistic characteristic of the useful and the noise signals.

2. During vector approximation, linear dependence between different components of electromagnetic field is used. Such an approach was applied in magnetotelluric and magnetovariational methods.

\section{Field scalar approximation}

The dependence of dipole radiation amplitude on the distance to a source during free space propagation has the following form:

$$
A=A^{0} \frac{\exp (-i f r / a)}{r}\left(\left(\frac{a}{f r}\right)^{2}+i \frac{a}{f r}-1\right) \sin (\theta)
$$

where $a=\frac{c}{2 \pi \sqrt{\epsilon \mu}} \approx 5 \cdot 10^{9} \mathrm{~m} / \mathrm{s}$-is the constant

$c, \epsilon, \mu$-is light velocity, electric and magnetic susceptibility of a medium,

$A^{0}$ - is the parameter depending on dipole moment, medium parameters and radiation frequency,

$\theta$-is the angle between radiation propagation direction and dipole moment,

$f, r-$ are the frequency and distance, respectively.

Consequently, the field amplitude in the near zone is proportional to $r^{-3}$, and in the far zone it is proportional to $r^{-1}$. Thus, the natural field in a seismically active zone may approximately be considered as a sum of the far (atmospheric-lightning) $E_{L}$ and near (lithospheric) $E_{G}$ fields:

$$
E \approx E_{G}+E_{L}=\frac{E^{0}{ }_{L}}{r_{G}}+E_{L}^{0} \frac{a^{2}}{f^{2} r_{L}^{3}}
$$


where $E_{L}{ }^{0}, E^{0}{ }_{G}$ are the effective amplitudes of lightning and lithospheric sources at the place of generation, $r_{L}, r_{G}$-is the distance from a detector to lightning and lithospheric sources.

To investigate signals effectively, it is necessary for a useful signal to exceed the noises $\frac{E_{G}}{E_{L}}>1$. Unfortunately, in our case this condition is not satisfied. Development of the method for registration and detection of signals from lithospheric sources reduces to the search for such a measurement procedure of field $F(E)$ parameters which would allow us to satisfy the inequality

$$
\frac{F\left(E_{G}\right)}{F\left(E_{L}\right)}>1
$$

In expression (3), the numerator and denominator function arguments in the left part of the inequality have different degrees $x$. Thus, spatial derivative may be used as such a function. It may be quite easily realized in the form of a measurement process.

We introduce an auxiliary distance in the detector vicinity which satisfies the conditions $x<<$ $r_{L}, x \backsim r_{G}$. Then the relation (3) may be written as follows

$$
\frac{d}{d x}\left(\frac{E_{G}^{0}}{r_{G}+x}\right) / \frac{d}{d x}\left(\frac{E^{0}{ }_{L} a^{2}}{f^{2}\left(r_{L}+x\right)^{3}}\right)>1
$$

After differentiation of (4) at $x=0$ it has the following form

$$
\frac{E^{0}{ }_{G} f^{2} r_{L}^{4}}{3 a^{2} E_{L}{ }^{0} r_{G}^{2}}>1
$$

Estimates of this inequality in the frequency range of $f \backsim 1 \div 10^{3} \mathrm{~Hz}$ at the distances to the center of lightning activity of megameter order of $\left(r_{G} \sim 10^{3} \mathrm{~m}\right)$, air breakdown intensity during a lightning stroke of $E_{L}{ }^{0} \sim 10^{6} \mathrm{~V} / \mathrm{m}$, field intensity induced by dislocational motion in a rock of $E_{G 0} \sim 10^{-10} \div 10^{-7} \mathrm{v} / \mathrm{m}[19,20,21]$, show that this inequality is fulfilled more than enough. In other words, in the frequency range of $f \sim 1 \div 10^{3} \mathrm{~Hz}$ a signal from a lithospheric source may be definitely registered and distinguished from strong noise of atmospheric-lightning orignin applying an electromagnetic sensor based on registration of electomagnetic field spatial derivative. We should note that both the signal of lithospheric emission and the signal of induction origin caused by the currents induced by the field of remote sources (near equatorial lightning) may be distinguished by such a way in geological bodies contrast in electrical conductivity. The initial signal causing inductive currents is rejected. The practical realisation of such a sensor is a quadrupole antenna. It is a system of two spaced identical dipole antennas with oppositely oriented dipole moments $[4,5]$.

\section{Properties of well-sensor}

The conditions of wave propagation in the Earth crust get better with depth and vice-versa when approaching to the surface. That is why to investigate the lithosphere wave fields more effectively, it is necessary to receive signals from as great depth as possible. Such approach is possible in mines or in wells covering large territories and diversity of geological conditions.

From the radio physical point of view, a well is 1 . a waveguide of longitudinal acoustic and electromagnetic vibrations; 2 . a converter of transverse electromagnetic and acoustic vibrations into longitudinal vibrations; 3 . a collector of acoustic and electromagnetic longitudinal vibrations of deep layers; 4. a resonance structure. A well casing is a magnetic circuit allowing us to get the magnetic component of electromagnetic emission on the surface. Moreover, it is a magnetoelastic converter of acoustic vibrations into electromagnetic ones (Villary effect). It is a good conductor buried into a 
poorly conducting environment of a rock. Electric field vertical component generates vertical currents in the pipe material, which in their turn generate a magnetic vortex with an axis along the pipe casing axis.

\section{Data processing for asymmetrical quadrupole}

Application of wells in compensative reception by a pair of antennas causes additional difficulties since one of the antennas has a steel casing pipe of the well as a ferrite core which is also a magnetic circuit for channeling of electromagnetic vibration magnetic component to the surface. The second antenna registers only noise signal which is used to detect the useful lithospheric signal. It is difficult to make the amplitude-frequency characteristics of these antennas identical for qualitative compensation of noise signal. Thus, it is necessary to make independent signal registration by each antenna and the compensation should be carried out at the stage of preliminary processing. In order to do that, a weighting function is introduced during the processing. It allows us to compensate the difference of amplitude-frequency characteristics of the antennas. The power spectra of mixture signal and the compensating signal are: $J_{0}(\omega)=\left|\tilde{F}\left(I_{0}\right)^{2}\right|, J_{k}(\omega)=\left|\tilde{F}\left(I_{k}\right)^{2}\right|$. Here $J,|\tilde{F}|, I_{0}, I_{k}$ are power spectrum, Fourier transform module, mixture signal and compensating signal, respectively. As long as the lithospheric signal is much weaker than the noise one, we may find the weighting coefficient in the assumption that the average power spectra of the mixture signal and the compensating signal should be equal with the accuracy to the weighting coefficients: $\widehat{F(\omega)} \cong a(\omega) \cdot\left(\widehat{I_{k}(\omega)}\right)$. Here the symbol $\widehat{R}$ denotes the average value of $R$.

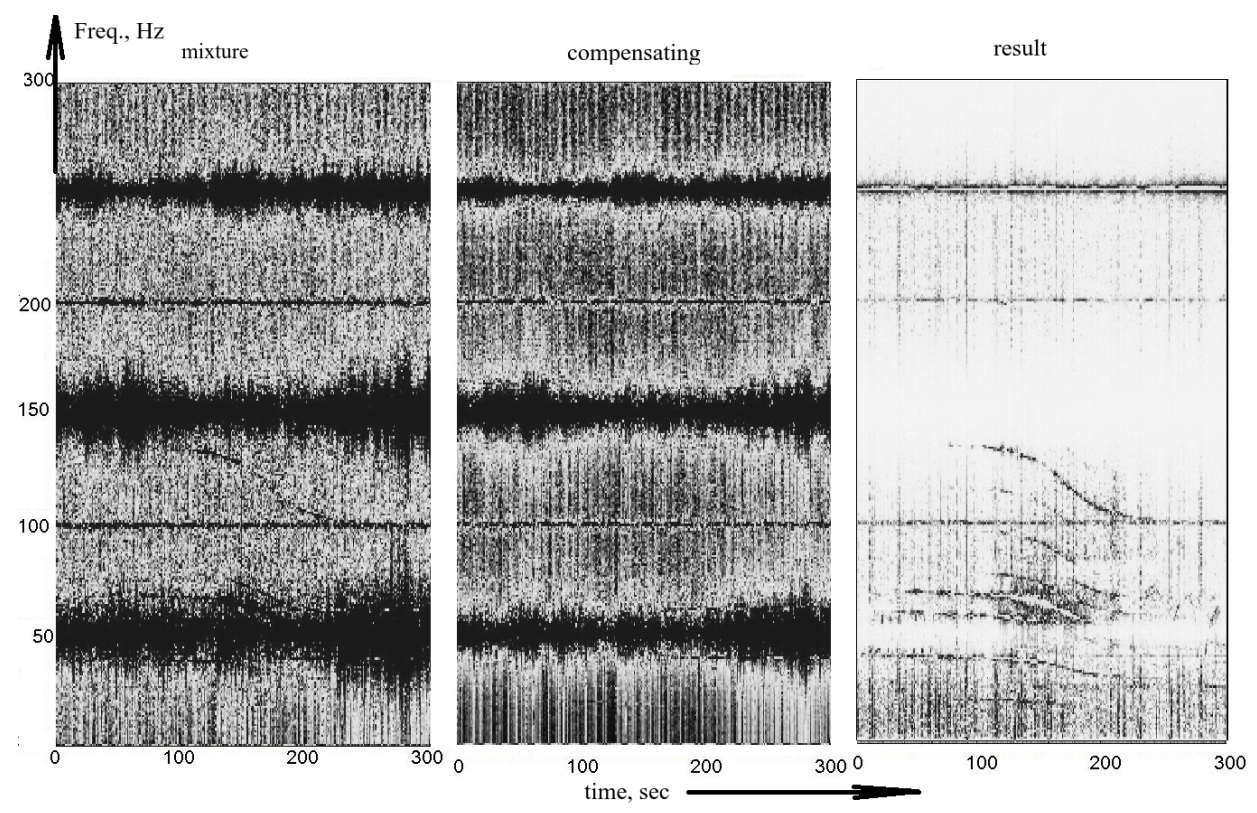

Figure 1. Signal power spectra. A mixture signal is to the left, the compensating signal is in the center, the result is to the right 
In order to find the weighting function $a(\omega)$, we apply the least square technique choosing $F(\omega)=$ $\left(I_{0}(\omega) \widehat{-a_{1}} \dot{I}_{k}(\omega)\right)^{2}$ as the efficiency function. Minimizing the efficiency function $\frac{\partial F(\omega)}{\left.\partial a_{1}(\omega)\right)}=0$, we

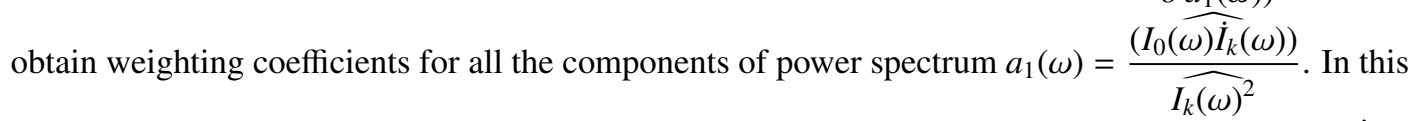
case the power spectrum of the denoised lithospheric signal has the form $I_{l}(\omega)=I(\omega)-a_{1}(\omega) I_{k}(\omega)$. Fig. 1 illustrates an example of power spectrum of a mixture, compensating signal and the result of detection of a lithospheric signal.

\section{Conclusions}

Manifestation of seismic events in electromagnetic field discovered at the end of the latest century has become the basis for the development of a new branch of knowledge, seismoelectromagnetism. Electromagnetic phenomena accompanying earthquakes occur in a wide frequency range. The most noticeable manifestations are observed in SLF-VLF range. The complexity of this phenomenon determined by strong effects of the main types of natural radiation, lightning stroke radiation, may significantly or completely be eliminated applying the approaches and methods described in this paper.

\section{References}

[1] V. N. Uvarov, Geofizicheskiy zhurnal, 34:6, 133-146 (2012) (in Russian)

[2] G. S. Golitsyn (eds.), Geofizika (Mir, Moscow, 1964), 500 (in Rissian)

[3] G. E. Yakovitskaya, Metody i tekhnicheskie sredstva diagnostiki kriticheskikh sostoyaniy gornykh porod na osnove elektromagnitnoy emissii, (Parallel', Novosibirsk, 2008), 315 (in Russian)

[4] V. N. Uvarov, G. I. Druzhin, D. V. Sannikov, Pribory i tekhnika eksperimenta, 26, 131-137 (2010)

[5] V.N. Uvarov, G.I. Druzhin, V.M. Pukhov, D.V. Sannikov, Technique for passive location of closely located sources of electromagnetic radiation at the background of powerful radiation of remote sources, Patent of RF G01V 3/08 No. 2473101, 30.06.2011. 